\title{
Integration of glass micropipettes with a 3D printed aligner for microfluidic flow cytometer
}

\author{
Abdullah Bayram ${ }^{a}$, Murat Serhatlioglu ${ }^{\mathrm{b}}$, Bulend Ortac ${ }^{\mathrm{b}}$, Serafettin Demic ${ }^{\mathrm{a}}$, \\ Caglar Elbuken ${ }^{\mathrm{b}}$, Mustafa Sen ${ }^{\mathrm{c}, *}$, Mehmet Ertugrul Solmaz ${ }^{\mathrm{d}, *}$ \\ a Department of Material Science and Engineering, Izmir Katip Celebi University, Izmir, Turkey \\ ${ }^{\mathrm{b}}$ UNAM - National Nanotechnology Research Center, Institute of Materials Science and Nanotechnology, Bilkent University, 06800 Ankara, Turkey \\ ${ }^{\mathrm{c}}$ Department of Biomedical Engineering, Izmir Katip Celebi University, Izmir, Turkey \\ ${ }^{\mathrm{d}}$ Department of Electrical and Electronics Engineering, Izmir Katip Celebi University, Izmir, Turkey
}

\section{A R T I C L E I N F O}

\section{Article history:}

Received 10 July 2017

Received in revised form

30 November 2017

Accepted 30 November 2017

Available online 2 December 2017

\section{Keywords:}

Flow cytometry

Hydrodynamic focusing

Micropipette

3D printing

Optofluidics

\begin{abstract}
A B S T R A C T
In this study, a facile strategy for fabricating a microfluidic flow cytometer using two glass micropipettes with different sizes and a 3D printed millifluidic aligner was presented. Particle confinement was achieved by hydrodynamic focusing using a single sample and sheath flow. Device performance was extracted using the forward and side-scattered optical signals obtained using fiber-coupled laser and photodetectors. The 3-D printing assisted glass capillary microfluidic device is ultra-low-cost, not labor-intensive and takes less than 10 min to fabricate. The present device offers a great alternative to conventional benchtop flow cytometers in terms of optofluidic configuration.
\end{abstract}

(c) 2017 Elsevier B.V. All rights reserved.

\section{Introduction}

Flow cytometry has proven to be a sensitive, quantitative and non-invasive approach to obtain valuable information from single cells [1-4]. It is routinely used in clinical laboratories for the identification and classification of certain cancers, such as leukemia [5-7] and lymphoma [8,9], and even for the diagnosis of life-threatening diseases such as AIDS $[10,11]$. Because of the disadvantages of the conventional benchtop flow cytometers such as high cost, large size, maintenance, complex configuration and the need for large volumes of reagents, a great deal of attention has been paid to fabricate microfluidic devices using microfabrication technologies in recent years. Microfluidic devices offer many advantages like simplicity, low cost, low consumption of reagents, rapid analysis, small size and highly controllable environment for particle manipulation. Focusing in flow cytometers is used to confine cells close to the center of the channel so that they can pass through the optical interrogation area one at a time, a phenomenon that improves sin-

\footnotetext{
* Corresponding authors.

E-mail addresses: mustafa.sen@ikc.edu.tr (M. Sen), mehmete.solmaz@ikc.edu.tr (M.E. Solmaz)
}

gle cell analysis and limits sample adsorption to the channel wall. A variety of focusing methods exploiting different physical mechanisms have been developed; hydrodynamic focusing [12-15], dielectrophoresis [16,17], acoustics [18-21] and electroosmosis [22-24]. Hydrodynamic focusing enables focusing in a wide velocity range and has no special requirement for particles, cells and fluids used. It is usually used to guide particles or cells in a confined space by manipulating the streamlines. Unlike 2D hydrodynamic focusing that focuses in only one lateral dimension, confining the stream of particles in 3D ensures the uniform flow of particles along the detection area. 3D hydrodynamic focusing improves a number of features of the microfluidic flow cytometers including performance, accuracy, stability, sensitivity and detection resolution $[15,25,26]$. Up to date, different 3D focusing strategies have been proposed to fabricate microfluidic devices. For example, Sundararajan et al. used six sheath inlets and a five-layer design for 3D focusing of sample fluid in flow [27]. However, the use of multiple syringes and multiple layer fabrication made the design rather complicated. Gorthi et al. fabricated a PDMS based single-layer microfluidic device that consists of three inlets (one for sample flow and two for sheath flow) and an outlet to realize hydrodynamic 3D flow focusing [12]. Although PDMS device fabrication involved a single layer, the master required for replica molding 
and device fabrication required multilayer fabrication by means of photolithography. Testa et al. developed a micro-flow cytometer capable of self-aligned 3D hydrodynamic focusing [13]. To make the device, the design was first printed on two $3 \mathrm{~mm}$-thick polymethylmethacrylates (PMMA) using a high precision computer numerical control micromilling machine followed by solvent assisted thermal bonding. Agrawal et al. proposed a new strategy with intricate microfabrication utilizing two bends of opposite curvature, single sheath inlets and exploiting secondary forces and centrifugal effects for 3D hydrodynamic focusing of dye, particles and cells [15]. Recently, femtosecond laser micromachining was presented as a new potential fabrication tool for making microfluidic networks that implement hydrodynamic focusing with two inlets; one for the sample and one for the sheath flow [14,28].3D printing technology has also been employed to make complex microfluidic devices out of discrete elements with less effort [29-31]. 3D printing still lacks the sufficient patterning resolution required for fabricating micron scaled fluidic devices with good alignment accuracy [32]. The majority of reported microfluidic flow cytometers are either too complex or require multiple sheath flows to realize 3D focusing and further research is needed to make less labor intensive and cost-effective microfluidic flow cytometers capable of efficient 3D hydrodynamic focusing.

In this proof of concept study, a facile strategy for making a microfluidic flow cytometer by using two glass micropipettes with different size and a 3D printed millifluidic aligner was demonstrated. Micropipettes have already been introduced to microfluidics; for example, Weitz et al. used micropipettes for creating monodisperse microdroplets in single or multiple-emulsion forms [33,34]. The fabrication of micropipettes from glass capillaries using a micropuller is relatively easy with minimal fabrication time, and the dimensions of micropipette tip opening can be easily manipulated by either changing the pulling parameters or grinding the tip with a microgrinder [35-37]. To the best of our knowledge, this is the first study that combines 3D printing with micropipette technology for efficient hydrodynamic focusing of particles. The proposed fabrication strategy is facile, straightforward and ultralow-cost as it takes less than one dollar to fabricate the optofluidic device.

\section{Materials and methods}

\subsection{Materials}

Glass capillary with filament (O.D./I.D.: 1/0.6 mm; length: $90 \mathrm{~mm}$ )(Narishige, Japan), blood gas capillary tubes (O.D./I.D.: 2.3/1.85 mm; length: $125 \mathrm{~mm}$ ) (Marianfeld, Germany), polystyrene microbeads with a diameter of $5.95 \pm 0.12 \mu \mathrm{m}$ (Polysciences, Inc., USA), phosphate-buffered saline (PBS) (Sigma Aldrich, USA), sealant (Pattex, Henkel, Germany), a high temperature mold material (HTM140 M, EnvisionTEC, USA), a single mode (SM) blue laser illumination fiber (LP405-SF10, Thorlabs, USA), forward scatter (FSC) and side scatter (SSC) fibers (M43L01, Thorlabs, USA).

\subsection{Device fabrication}

First, a small glass capillary tube (O.D./I.D.: 1/0.6 mm; length: $90 \mathrm{~mm}$ ) was pulled using a micropuller (PC-10 Micropulling Machine, Narishige, Japan) to produce two microneedles with the following parameters; option 2, first-pull position adjustment plate: $6 \mathrm{~mm}$, second-pull position adjustment plate: $2 \mathrm{~mm}$, one type light and one type heavy weights, no. 1 heater level: 65 and no. 2 heater level: 37 [35]. The tip of the small micropipette was grinded to have a tip opening inner diameter of approximately $150 \mu \mathrm{m}$ using a microgrinder (EG-401, Narishige, Japan) (Fig. 1 $\mathrm{A}_{\mathrm{I}}$ ).
The desired tip opening diameter was adjusted using the ocular micrometer of the microgrinder. Then, a large glass capillary tube (O.D./I.D.: 2.3/1.85 mm; length: $125 \mathrm{~mm}$ ) was pulled the same way as the small capillary tube using the following parameters; option 1, two type light and two type heavy weights, no. 1 heater level: 75 . The large micropipette was pulled in a way to have a microchannel with an approximately $90 \mu \mathrm{m}$ inner diameter and a length of no less than $10 \mathrm{~cm}$. The tip of the large micropipette was grinded only to measure the tip opening diameter properly (Fig. $1 \mathrm{~A}_{\mathrm{II}}$ ). Both micropipettes were cleaned thoroughly in distilled water using ultrasonic before use. The small micropipette was centerpositioned inside the large micropipette using a micropipette aligner (Fig. $1 A_{\text {III }}, B_{I}$ ) a process that took only a few minutes. The micropipette aligner was first designed using a 3D CAD design software (SolidWorks 2016, USA) (Fig. S1A) and then produced from a high temperature mold material (HTM140 M) using a 3D rapid prototype manufacturing system (Perfactory 4 Mini with ERM, EnvisionTEC, USA) with high precision. As for the assembly of the device, the large micropipette was first inserted in the corresponding hole of the aligner and a sealant was used to both fix the micropipette and prevent any leakage from the small gap between the capillary and the aligner hole. Then, the small micropipette was inserted into the corresponding hole of the aligner to position the small micropipette inside the large one under a stereo microscope and the same sealant as before was used for micropipette fixation and leakage prevention (Fig. S1B). Prior to use, all devices were checked for leakage by pumping water from the two inlets at pressures up to 2 bars, which is the upper limit the pressure pump can provide.

\subsection{Device characterization}

The 3D hydrodynamic focusing ability of the device was investigated using deionized water (DI) colored with a blue food dye and colorless PBS as sample and sheath solutions, respectively. A pressure pump (Elveflow, OB1) was used to pass the solutions through the micropipettes. In this setting, the PBS solution from the large micropipette hydrodynamically focused the blue color solution introduced from the small micropipette by completely surrounding it (Fig. $\left.1 \mathrm{~B}_{\mathrm{II}}\right)$. The impact of the ratio between sheath and sample flow pressures on the 3D hydrodynamic focusing was investigated in a range from 0.96 to 1.064 (sheath/sample flow pressures) (Fig. 2A). The 3D hydrodynamic focusing was recorded using a camera (Basler Ace acA2040-90uc, Basler AG, Germany) attached to a microscope (Krüss MSL4000, Germany).

Next, the device was tested with microparticles of uniform size using an optical test system. The test system consisted of the following components; a single mode (SM) blue laser illumination fiber, forward scatter (FSC) and side scatter (SSC) fibers, two photo diodes (FSC and SSC) (DET02AFC, Thorlabs, USA), a $405 \mathrm{~nm}$ laser source (LP405-SF10, Thorlabs, USA), a pressure pump and an oscilloscope (Tektronix, MDO3104) (Fig. 3A and Fig. S2A). In addition, a flat PMMA plate with fiber guidance grooves was used to position all the fibers (Fig. S2B and C). An adhesive tape was used to fix the fibers in the grooves carved on the plate by $\mathrm{CO}_{2}$ laser (Epilog Zing 30W, Epilog Laser, USA). Basically, the laser illumination fiber with a diameter of $125 \mu \mathrm{m}$ was positioned perpendicular $\left(90^{\circ}\right)$ to the microchannel where the microchannel diameter and the width of the focused sample solution were $\sim 130$ and $\sim 18 \mu \mathrm{m}$, respectively. The optical fiber with the following specifications (numerical aperture (NA): 0.12 , mode field diameter (MFD): $3.6 \pm 0.5 \mu \mathrm{m}$ and center wavelength: $405 \mathrm{~nm}$ ) was used to translate a single mode (SM) laser to the interrogation zone where the beam diameter calculated with Gaussian beam approximation is $11.48 \mu \mathrm{m}$. To collect the scattered light through the photo detector, FSC and SSC fibers were positioned with angles of $167^{\circ}$ and $210^{\circ}$ to the laser illumina- 
A)

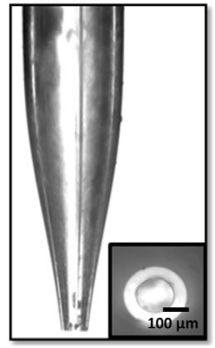

$\left.B_{1}\right)$

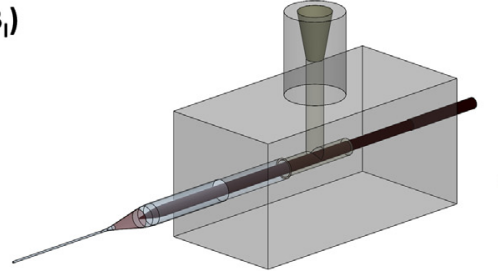

$\left.A_{11}\right)$

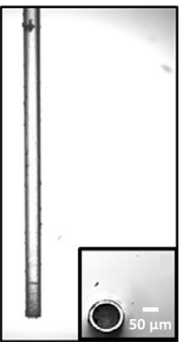

$A_{\text {III }}$ ii)

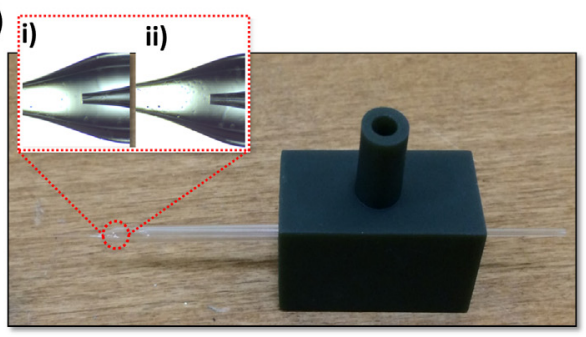

$\left.\mathbf{B}_{\|}\right)$

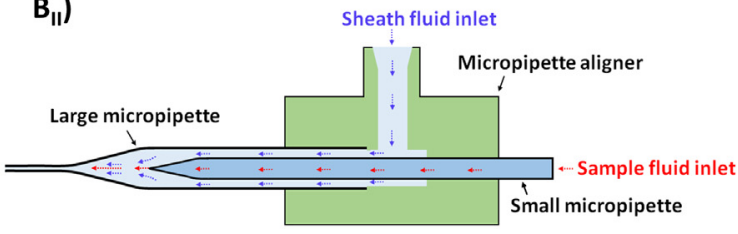

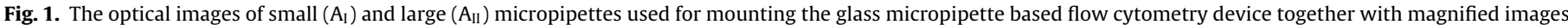

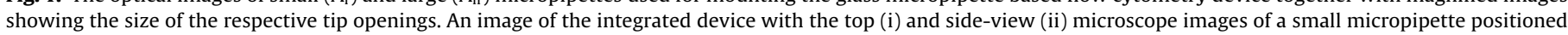

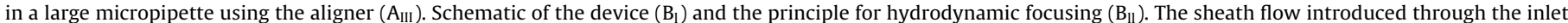
of the 3D-printed aligner focuses the sample fluid introduced from the small micropipette.

\section{A)}
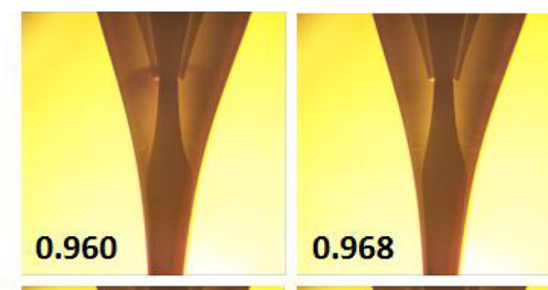

0.976
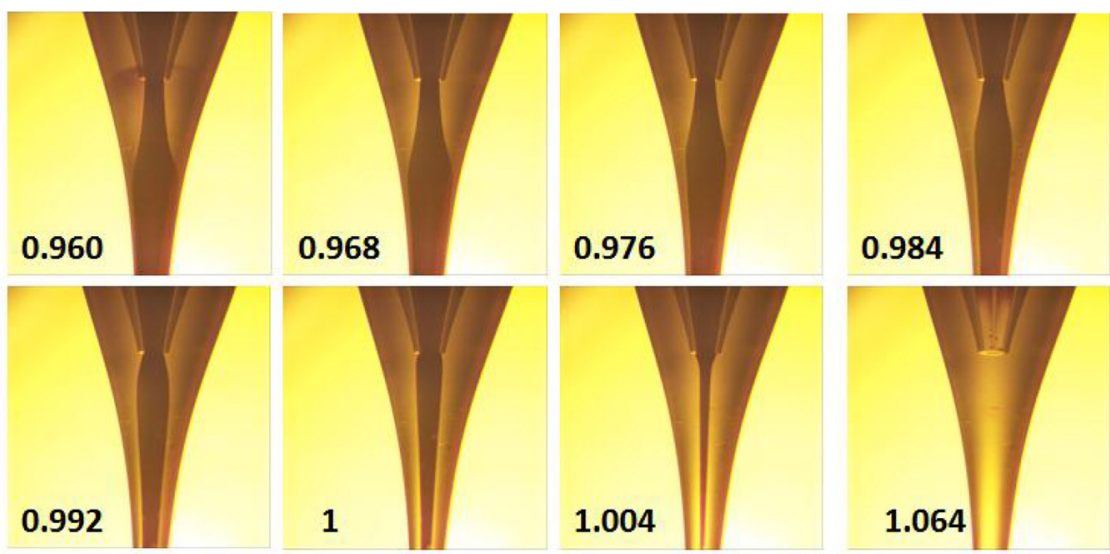

1

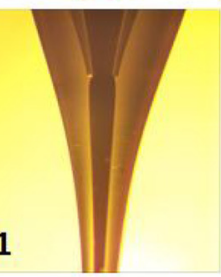

B)

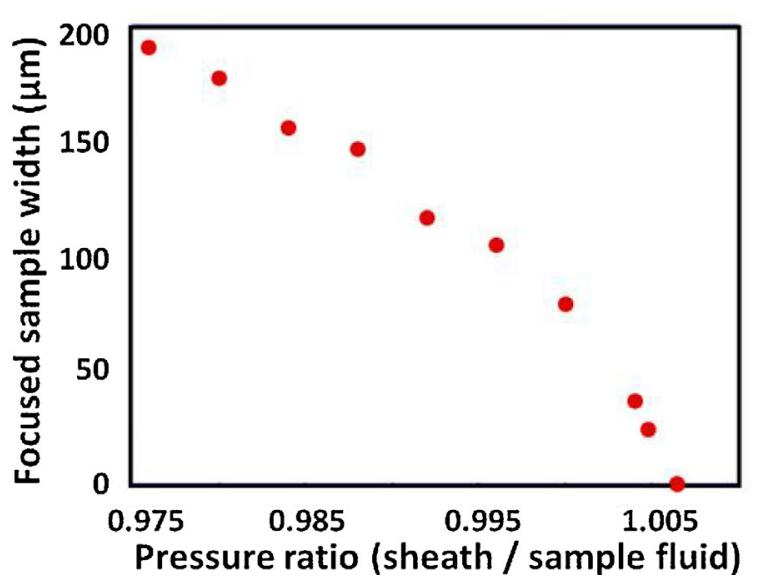

$\left.C_{1}\right)$

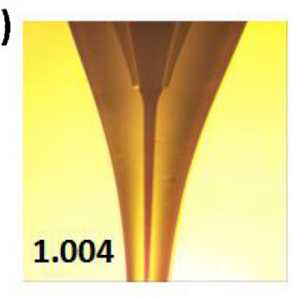

$\left.\mathrm{c}_{111}\right)$

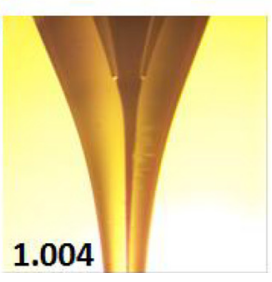

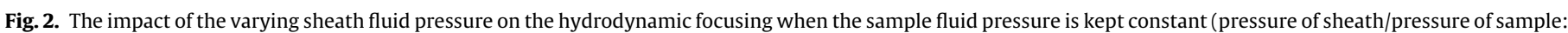

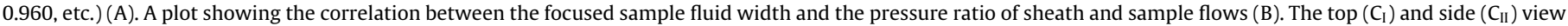
microscope images of 3D focusing achieved at a ratio of 1.004 between sheath and sample flow pressures.

tion fiber, respectively. PBS and polystyrene microbeads containing PBS solutions were used as sheath and sample solutions, respectively. Prior to use, the microparticle (diameter: $5.95 \pm 0.12 \mu \mathrm{m}$ ) stock solution was diluted in a mixture (PBS solution + blue food dye) filtered using $0.2 \mu \mathrm{m}$ pore syringe filter to realize a final concentration of $\sim 1000$ particles/ $\mu$ l. The blue color mixture was used simply for better visualization. The microparticle containing suspension was also sonicated for $15 \mathrm{~min}$ to minimize aggregation during experiments. Pressure pump was used to pass PBS (sheath) and polystyrene microsphere containing suspension through the large and small micropipettes at a sheath/sample solution pressure 
A)

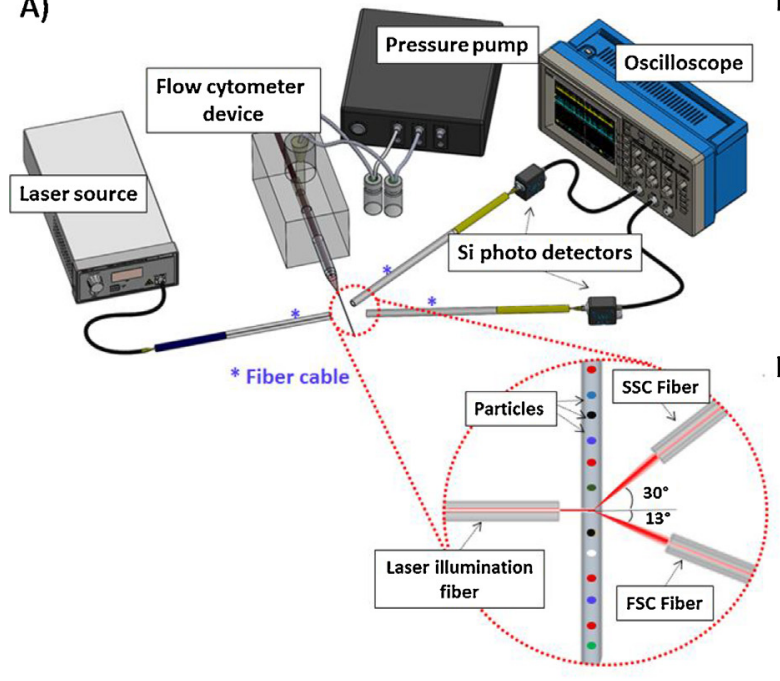

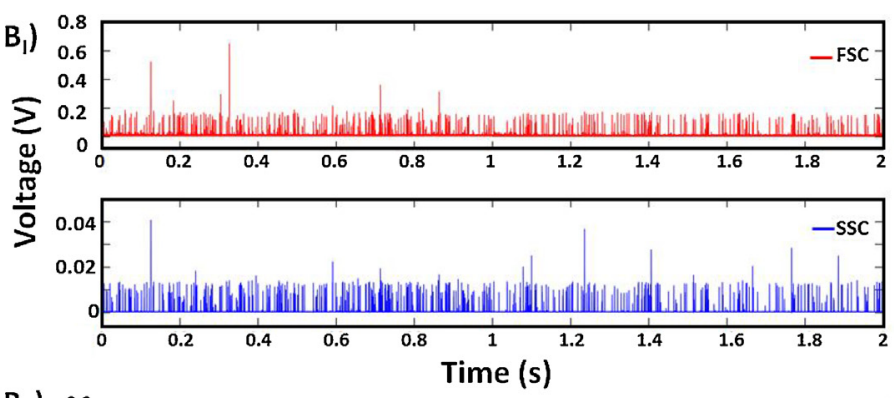

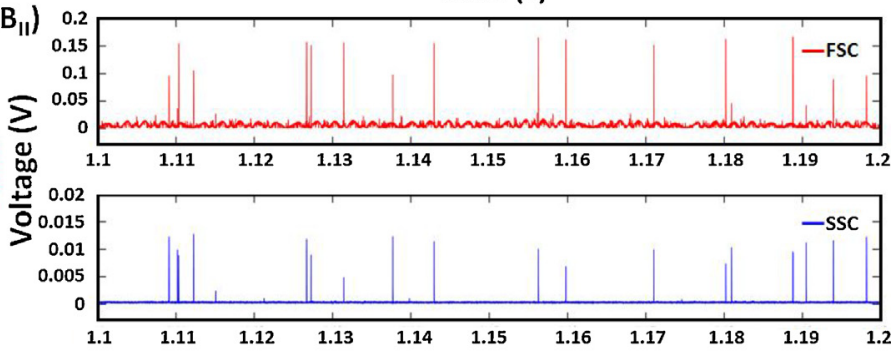

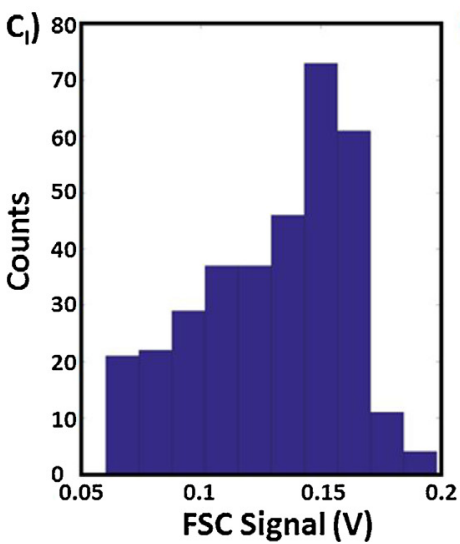
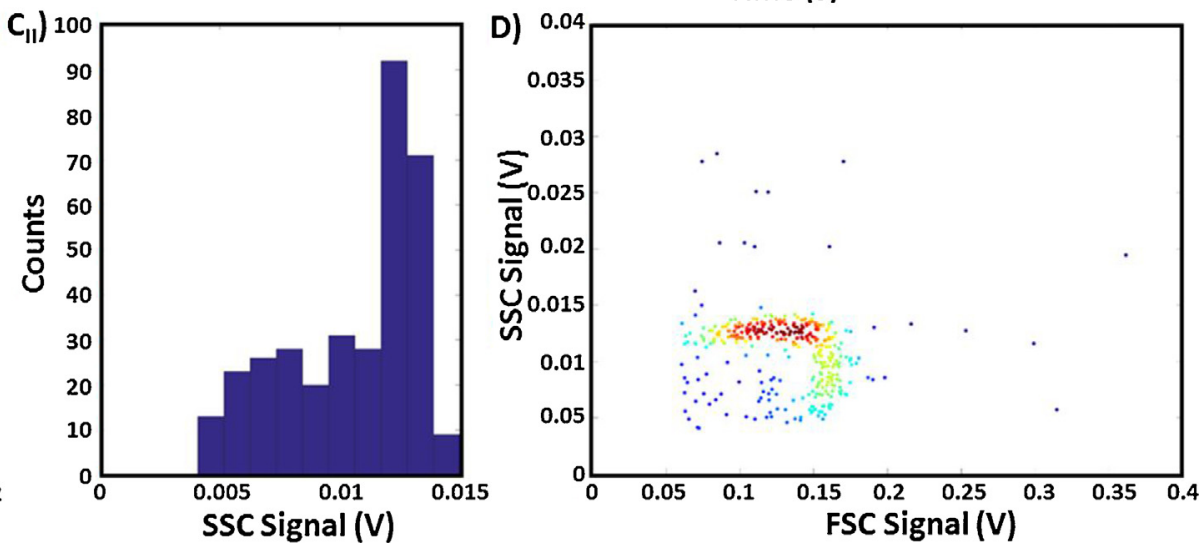

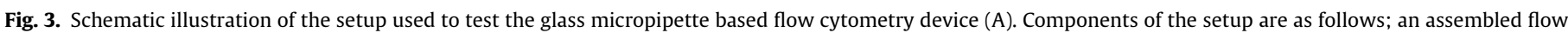

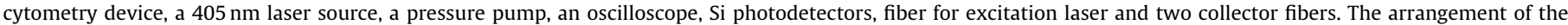

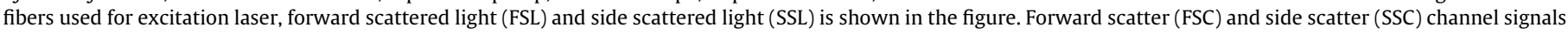

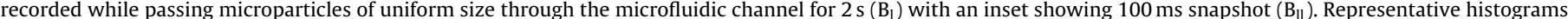

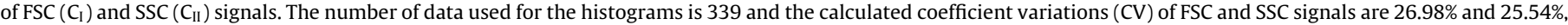
respectively. FSC-SSC scatter plot obtained with microparticles of uniform size (D).

ratio of 1.004 . The oscilloscope was used to quantify the voltage signals from the photodetectors at a $0.5 \mathrm{MHz}$ sampling rate.

\section{Results and discussions}

The design of the present microfluidic flow cytometer device is quite simple and easily comprehensible (Fig. $1 \mathrm{~B}_{\mathrm{I}-\mathrm{II}}$ ). A complete device is composed of three components; a micropipette aligner, a small and a large micropipettes (Fig. $1 A_{\text {III }}$ ). Precise fabrication of aligner holes is important for successful micropipette alignment. Manufacturer reports that HTM140 is very durable and high precision material designed to withstand both the heat and pressure of vulcanizing a model with great detail and no loss of dimensional stability. Given the design dimensions of the model in Fig. S1A, the average diameters of small and large holes of three different aligners were measured to be $1049.9 \pm 8.1 \mu \mathrm{m}$ and $2303.5 \pm 8.9 \mu \mathrm{m}$, respectively (Fig. S3A, B). It is safe to say that the $3 \mathrm{D}$ rapid prototype manufacturing system along with HTM140 material provides dimension accuracy for precise alignment of micropipettes. The assembly of the device took less than 10 min including the time needed to grind the tip of the small micropipette. The micropipette aligners can be retrieved easily and used repeatedly for fabrication of a new microfluidic flow cytometer device. The only consum- ables used to fabricate a new device are the sealant and the glass capillaries, which makes the device ultra-low-cost as both of the consumables cost less than one dollar.

The 3D hydrodynamic focusing ability of the device was demonstrated using deionized water (DI) colored with a blue food dye and phosphate buffer saline (PBS) as sample and sheath solutions, respectively. The different colors of the two solutions made it possible to easily observe the confinement of the sample solution in the microchannel of the large micropipette (Fig. 2A). Hydrophilic surface is important and desirable for microfluidic systems to achieve various functions such as facilitating fluid flow into the microchannels [38]. The water contact angle test result showed that HTM140 is hydrophilic with an average contact angle value of $37.1^{\circ}$ (Fig. S4). Using the3D printed micropipette aligner with easy-to-operate two-inlet configuration; the sheath solution completely surrounds and confines the sample solution in a highly efficient manner (Figs. $1 A_{I I}$ and $2 \mathrm{~A}$ ). The results showed that the microchannel was filled with the blue color solution at the ratio of 0.96 . When the ratio was raised to 1.064 , the flow through the small micropipette was blocked by the sheath solution. The small difference between the two ratios demonstrates that the pressure of the two fluids must be controlled precisely for an efficient 3D focusing. The relationship between the width of the hydrodynamically 
focused sample and the pressure ratio between sheath and sample flows is given in Fig. 2B. The width of the sample solution was measured approximately $1.53 \mathrm{~mm}$ away from the tip of the small micropipette (Fig. S5). The relationship appears to be almost linear, which suggests that the width of the 3D focused sample solution can be precisely controlled by the ratio between sheath and sample flow pressures. In addition, side view microscope images of the microchannel were taken both horizontally and vertically at a ratio of 1.004 (sheath/sample flow pressure) to see the focused flow and thus verify the 3D hydrodynamic focusing with the present device (Fig. 2- $\mathrm{C}_{\mathrm{I}-\mathrm{II}}$ ). Both images showed that the sample flow was focused close to the center of the microchannel.

Next, the performance of the microfluidic flow cytometer device in 3D hydrodynamic focusing of microparticles was evaluated using an optical test system. Without any particle flow, the FSC and SSC signals show DC offset values due to collecting some amount of light. Fig. $3 \mathrm{~B}_{\mathrm{I}}$ shows 2-s FSC and SSC channel signals recorded while microparticles of uniform size were passing through the microfluidic channel. Each peak observed in Fig. $3 \mathrm{~B}_{\mathrm{I}-\mathrm{II}}$ corresponds to a microparticle passing by the interrogation point. From to the data with $\sim 170$ events/s throughput, an FSC peak was recorded for each SSC signal, which indirectly proves that all of the microparticles were focused properly and passed through the laser interrogation region at the same point along the microchannel. Fig. $3 \mathrm{~B}_{\mathrm{II}}$ shows a close-up view of peak events for $100 \mathrm{~ms}$. Since the events were well separated, there is a great possibility to further increase the throughput. The signals were post-processed with MATLAB to obtain the number, peak values and positions of the events. Gating was applied to improve the population distribution with common properties as seen in commercial flow cytometers and to avoid multi-particle events such as aggregated particles. Fig. $3 \mathrm{C}_{\mathrm{I}-\mathrm{II}}$ shows the histograms of the peaks observed in FSC and SSC signals given in Fig. $3 \mathrm{~B}_{\mathrm{I}}$, respectively. The calculated coefficient of variation (CV) values obtained for FSC and SSC signals were $26.98 \%$ and $25.54 \%$, respectively. Lower $\mathrm{CV}$ values could be achieved by adjusting the sheath and small solution pressures more precisely [39]. Accurate alignment of the illumination fiber and FSC/SSC fibers is critical. In this setup, these fibers were aligned using guideline grooves carved on a PMMA substrate by $\mathrm{CO}_{2}$ laser (Fig. $\mathrm{S} \mathrm{A}_{\mathrm{I}-\mathrm{II}}$ ). In other words, the illumination and FSC/SSC fibers were aligned automatically once they were fixed on the plate using a tape. However, the alignment between these fibers and glass micropipette was done manually using an XYZ stage (Fig. S6B and C). This might be a limitation for the current system as it might be time-consuming for researchers who are not familiar with such instruments. To overcome this limitation, a new strategy will be developed to bypass the manual alignment stage. Another limitation of the current setup is sample recovery. This issue will also be addressed in the future by collecting the outgoing sample in a channel with minimal dilution. A cell sorting mechanism may also be integrated into the system to divert cells of interest. Scatter plot in Fig. 3D depicts the correlation between FSC and SSC signals for $2 \mathrm{~s}$ time duration. It is obvious that most of the events were gathered in a small region, a result that confirms the proper functioning of the device. As compared to commercial state-of-the-art optical flow cytometers, bulk optics (laser diodes, optical lenses, mirrors, filters etc.) and mechanical holders/aligners are excluded from the present setup. The present setup uses fiber optics, which provides great deal of flexibility when considering the integration and stability of optical elements.

\section{Conclusion}

In conclusion, the fabrication of a low-cost and simple microflow cytometry device capable of hydrodynamic focusing was demonstrated using different size glass capillaries and a micropipette aligner printed with a 3D rapid prototyping manufacturing system. The assembly of the device took less than 10 min including micropipette tip grinding and the final device is ultra-low cost. The device showed an excellent performance in hydrodynamic focusing of DI water colored with a blue food dye. The device was also used for 3D hydrodynamic focusing of microparticles of uniform size where distinct FSC and SSC signals of microparticles passing through the optical interrogation point were successfully recorded. Given the separation between each event, the device has the potential for further increase in throughput. The device offers simplicity, low-cost and good performance in hydrodynamic focusing and has a great potential to be used in a wide range of flow cytometry applications.

\section{Appendix A. Supplementary data}

Supplementary material related to this article can be found, in the online version, at doi:https://doi.org/10.1016/j.sna.2017.11. 056

\section{References}

[1] S. Gawad, L. Schild, P. Renaud, Micromachined impedance spectroscopy flow cytometer for cell analysis and particle sizing, Lab Chip 1 (2001) 76-82.

[2] L. Wu, X. Wang, J. Zhang, T. Luan, E. Bouveret, X. Yan, Flow cytometric single-cell analysis for quantitative in vivo detection of protein-protein interactions via relative reporter protein expression measurement, Anal. Chem. 89 (2017) 2782-2789.

[3] A.K. Lehmann, S. Sornes, A. Halstensen, Phagocytosis: measurement by flow cytometry, J. Immunol. Methods 243 (2000) 229-242.

[4] M. Roederer, J.M. Brenchley, M.R. Betts, S.C. De Rosa, Flow cytometric analysis of vaccine responses: how many colors are enough? Clin. Immunol. 110 (2004) 199-205.

[5] E.G. Weir, M.J. Borowitz, Flow cytometry in the diagnosis of acute leukemia, Semin. Hematol. 38 (2001) 124-138.

[6] J.M. Peters, M.Q. Ansari, Multiparameter flow cytometry in the diagnosis and management of acute leukemia, Arch. Pathol. Lab. Med. 135 (2011) 44-54.

[7] P. Theunissen, E. Mejstrikova, L. Sedek, A.J. van der Sluijs-Gelling, G. Gaipa, M. Bartels, et al., Standardized flow cytometry for highly sensitive MRD measurements in B-cell acute lymphoblastic leukemia, Blood 129 (2017) 347-357.

[8] J.R. Fromm, A. Thomas, B.L. Wood, Flow cytometry can diagnose classical hodgkin lymphoma in lymph nodes with high sensitivity and specificity, Am. J. Clin. Pathol. 131 (2009) 322.

[9] I. Cozzolino, M. Rocco, G. Villani, M. Picardi, Lymph node fine-needle cytology of non-hodgkin lymphoma: diagnosis and classification by flow cytometry, Acta Cytol. 60 (2016) 302-314.

[10] I.C. Clift, Diagnostic flow cytometry and the AIDS pandemic, Lab. Med. 46 (2015) e59-e64.

[11] S.L. Waldrop, C.J. Pitcher, D.M. Peterson, V.C. Maino, L.J. Picker, Determination of antigen-specific memory/effector CD4+ T cell frequencies by flow cytometry: evidence for a novel, antigen-specific homeostatic mechanism in HIV-associated immunodeficiency, J. Clin. Invest. 99 (1997) 1739-1750.

[12] G. Eluru, L.A.N. Julius, S.S. Gorthi, Single-layer microfluidic device to realize hydrodynamic 3D flow focusing, Lab Chip 16 (2016) 4133-4141.

[13] G. Testa, G. Persichetti, R. Bernini, Micro flow cytometer with self-aligned 3D hydrodynamic focusing, Biomed. Opt. Express 6 (2015) 54-62.

[14] P. Paie, F. Bragheri, R.M. Vazquez, R. Osellame, Straightforward 3D hydrodynamic focusing in femtosecond laser fabricated microfluidic channels, Lab Chip 14 (2014) 1826-1833.

[15] S. Tripathi, A. Kumar, Y.V. Bala Varun Kumar, A. Agrawal, Three-dimensional hydrodynamic flow focusing of dye, particles and cells in a microfluidic device by employing two bends of opposite curvature, Microfluid. Nanofluid. 20 (2016) 34.

[16] H. Chu, I. Doh, Y.-H. Cho, A three-dimensional (3D) particle focusing channel using the positive dielectrophoresis ( $\mathrm{pDEP}$ ) guided by a dielectric structure between two planar electrodes, Lab Chip 9 (2009) 686-691.

[17] S. Yan, J. Zhang, M. Li, G. Alici, H. Du, R. Sluyter, et al., On-chip high-throughput manipulation of particles in a dielectrophoresis-active hydrophoretic focuser, Sci. Rep. 4 (2014) 5060.

[18] Y. Takahashi, A.I. Shevchuk, P. Novak, Y. Zhang, N. Ebejer, J.V. Macpherson, et al., Multifunctional nanoprobes for nanoscale chemical imaging and localized chemical delivery at surfaces and interfaces, Angew. Chem. Int. Ed. 50 (2011) 9638-9642.

[19] A.H. Yang, H.T. Soh, Acoustophoretic sorting of viable mammalian cells in a microfluidic device, Anal. Chem. 84 (2012) 10756-10762.

[20] Y. Chen, A.A. Nawaz, Y. Zhao, P.-H. Huang, J.P. McCoy, S.J. Levine, et al., Standing surface acoustic wave (SSAW)-based microfluidic cytometer, Lab Chip 14 (2014) 916-923. 
[21] P. Augustsson, J.T. Karlsen, H.-W. Su, H. Bruus, J. Voldman, Iso-acoustic focusing of cells for size-insensitive acousto-mechanical phenotyping, Nat. Commun. 7 (2016) 11556

[22] Y. Jia, Y. Ren, H. Jiang, Continuous-flow focusing of microparticles using induced-charge electroosmosis in a microfluidic device with 3D AgPDMS electrodes, RSC Adv. 5 (2015) 66602-66610.

[23] Z. Liu, A.J.H. Frijns, M.F.M. Speetjens, A.A. van Steenhoven, Particle focusing by AC electroosmosis with additional axial flow, Microfluid. Nanofluid. 18 (2015) 1115-1129.

[24] Y. Ren, J. Liu, W. Liu, Q. Lang, Y. Tao, Q. Hu, et al., Scaled particle focusing in a microfluidic device with asymmetric electrodes utilizing induced-charge electroosmosis, Lab Chip 16 (2016) 2803-2812.

[25] Z. Jingjing, Y. Zheng, Microfluidic hydrodynamic focusing for high-throughput applications, J. Micromech. Microeng. 25 (2015) 125006.

[26] A.A. Nawaz, X. Zhang, X. Mao, J. Rufo, S.C. Lin, F. Guo, et al., Sub-micrometer-precision, three-dimensional (3D) hydrodynamic focusing via "microfluidic drifting", Lab Chip 14 (2014) 415-423.

[27] N. Sundararajan, M.S. Pio, L.P. Lee, A.A. Berlin, Three-dimensional hydrodynamic focusing in polydimethylsiloxane (PDMS) microchannels, J. Microelectromech. Syst. 13 (2004) 559-567.

[28] M. Serhatlioglu, C. Elbuken, B. Ortac, M.E. Solmaz, Femtosecond laser fabrication of fiber based optofluidic platform for flow cytometry applications, Proc. SPIE 10058 (2017).

[29] J. Gottmann, M. Hermans, N. Repiev, J. Ortmann, Selective laser-induced etching of 3D precision quartz glass components for microfluidic applications-up-scaling of complexity and speed, Micromachines 8 (2017) 110.

[30] K.C. Bhargava, B. Thompson, N. Malmstadt, Discrete elements for 3D microfluidics, Proc. Natl. Acad. Sci. 111 (2014) 15013-15018.

[31] C.M.B. Ho, S.H. Ng, K.H.H. Li, Y.-J. Yoon, 3D printed microfluidics for biological applications, Lab Chip 15 (2015) 3627-3637.

[32] N. Bhattacharjee, A. Urrios, S. Kang, A. Folch, The upcoming 3D-printing revolution in microfluidics, Lab Chip 16 (2016) 1720-1742.

[33] A.S. Utada, E. Lorenceau, D.R. Link, P.D. Kaplan, H.A. Stone, D.A. Weitz Monodisperse double emulsions generated from a microcapillary device, Science 308 (2005) 537-541.

[34] S.H. Kim, J.W. Kim, J.C. Cho, D.A. Weitz, Double-emulsion drops with ultra-thin shells for capsule templates, Lab Chip 11 (2011) 3162-3166.

[35] M. Sen, A. Demirci, pH-dependent ionic-current-rectification in nanopipettes modified with glutaraldehyde cross-linked protein membranes, RSC Adv. 6 (2016) 86334-86339.

[36] M. Sen, K. Ino, H. Shiku, T. Matsue, A new electrochemical assay method for gene expression using HeLa cells with a secreted alkaline phosphatase (SEAP) reporter system, Biotechnol. Bioeng. 109 (2012) 2163-2167.
[37] M. Sen, Y. Takahashi, Y. Matsumae, Y. Horiguchi, A. Kumatani, K. Ino, et al, Improving the electrochemical imaging sensitivity of scanning electrochemical microscopy-scanning ion conductance microscopy by using electrochemical Pt deposition, Anal. Chem. 87 (2015) 3484-3489.

[38] J.M. Lee, M. Zhang, W.Y. Yeong, Characterization and evaluation of 3D printed microfluidic chip for cell processing, Microfluid. Nanofluid. 20 (2016) 5.

[39] C. Simonnet, A. Groisman, High-throughput and high-resolution flow cytometry in molded microfluidic devices, Anal. Chem. 78 (2006) 5653-5663.

\section{Biographies}

Abdullah Bayram received his B.Sc. in Physics from Izmir Institute of Technology in 2012 and his M.Sc. from the Department of Material Science and Engineering at Izmi Katip Celebi University in 2014. He is pursuing his Ph.D. at the same department.

Murat Serhatlioglu received a B.Sc. in Electrical and Electronics Engineering from Firat University in 2012. He is pursuing his Ph.D. at Bilkent University, Nationa Nanotechnology Research Center, Ankara, Turkey.

Dr. Bulend Ortac is an assistant professor at Bilkent University, National Nanotechnology Research Center, Ankara, Turkey.

Dr. Serafettin Demic is an associate professor at the Department of Material Science and Engineering, Izmir Katip Celebi University, Izmir, Turkey.

Dr. Caglar Elbuken is an assistant professor at Bilkent University, National Nanotechnology Research Center, Ankara, Turkey.

Dr. Mustafa Sen received his M.Sc. degree in Molecular Biology and Genetics from Istanbul Technical University in 2010 and $\mathrm{Ph}$. D degree in Bio-enginnering from Tohoku University in 2013. He currrently works as an assistant professor at the Department of Biomedical Engineering, Izmir Katip Celebi University. His main research focus is in the field of micro- and nanobiosensor development and their applications in single cell and micro-tissue analysis.

Dr. Mehmet E. Solmaz received his B.Sc. degree in Microelectronics from Sabanci University in 2004 and Ph.D. degree from Texas A\&M University in 2010. He was a part of Nanophotonics Research Group at University of Southern California during his postdoctoral research. Currently he is an associate professor in Electrica and Electronics Engineering Department of Izmir Katip Celebi University, Izmir Turkey. His research focuses on optical sensing applications using smartphones and microfluidic flow cytometry. 